\title{
Time-series photometric spot modeling
}

\section{The multi-periodic K5Ve binary V833 Tauri}

\author{
K. Oláh ${ }^{1}$, K. G. Strassmeier ${ }^{2,3}$, Zs. Kővári ${ }^{1}$, and E. F. Guinan ${ }^{4}$ \\ 1 Konkoly Observatory of the Hungarian Academy of Sciences, 1525 Budapest, Hungary \\ e-mail: kovari@konkoly.hu \\ 2 Astrophysical Institute Potsdam, An der Sternwarte 16, 14482 Potsdam, Germany \\ e-mail: KStrassmeier@aip.de \\ 3 Institute for Astronomy, University of Vienna, 1180 Vienna, Austria \\ 4 Dept. of Astronomy and Astrophysics, Villanova University, Villanova, PA 19085, USA \\ e-mail: edward.guinan@villanova.edu
}

Received 27 November 2000 / Accepted 7 March 2001

\begin{abstract}
We analysed all the existing photometric observations of V833 Tau and found long-term variability on time scales of $\approx 70$ years, 6.5 years and 2.4 years. Using $V$ and $I_{\mathrm{C}}$-band data from $1990-2000$, we obtained starspot surface distributions from 20 suitable light curves. We found that the spot-area changes generally follow the long-term light variations. Spot temperatures are determined for each of the datasets and an average value of $1230 \mathrm{~K} \pm 270 \mathrm{~K}$ relative to the photosphere is found. This value agrees with previous results from TiO modeling $(1330 \mathrm{~K} \pm 110 \mathrm{~K})$. Small scale spot temperature and area changes are recovered during 1997-1999, that we explain with a variable spot/faculae ratio. A powerful flare in $U B V(R I)_{\mathrm{C}}$ was observed in 1993 November. A $10000 \mathrm{~K}$ fit yielded a total emitted flare energy of more than $10^{34} \mathrm{ergs}$ from an area of about $1.3 \%$ of the stellar surface, $60 \%$ of it in the $V(R I)_{\mathrm{C}}$ bands. The flare characteristics are compared to another, 100-times smaller flare observed earlier in 1983.
\end{abstract}

Key words. stars: activity - stars: atmospheres - stars: late-type - stars: individual: V833 Tau - stars: binaries: close

\section{Introduction}

The history of the K2V active binary V833 Tau $\left(\mathrm{HD} 283750, V_{\max }=7^{\mathrm{m}} \cdot 90, B-V_{\max }=1 \cdot{ }^{\mathrm{m}} 01, P_{\text {orb }} \approx\right.$ $P_{\text {rot }} \approx 1$ d.79) until 1990 was summarized by Oláh \& Pettersen (1991). Basic physical properties on V833 Tau are given by Pettersen (1989) as $R / R_{\odot}=0.77$, $\log \left(L / L_{\odot}\right)=-0.68 \pm 0.1$, and $T_{\text {eff }}=4450 \mathrm{~K}$. Since then, the star has been the subject of a number of studies, mostly survey programs. At present the SIMBAD database lists 34 identifiers for this star.

Naftilan \& Fairchild (1993) found no evidence of lithium in V833 Tau, but in an earlier paper Pettersen (1989) reported the possible presence of lithium in its atmosphere. Later, lithium was confirmed by Barrado y Navascués et al. (1997) who measured a $16.2 \mathrm{~m} \AA$ equivalent width of the LiI 6707 line. Differing results were given by Saar et al. (1990), who found no variability in C IV and other high-temperature lines and also no variability in $\mathrm{H} \alpha$

Send offprint requests to: K. Oláh, e-mail: olah@konkoly.hu outside an observed flare, and by Strassmeier et al. (1990), who found strong and variable $\mathrm{H} \alpha$ emission measured on two consecutive nights during the same time interval when Saar et al.'s observations were taken, suggesting very short variability time scales (flare?). Later, Montes et al. (1995) reported slightly variable excess $\mathrm{H} \alpha$ emission. In a new survey, Montes et al. (1997) found moderate $\mathrm{H} \alpha$ emission including small variable self-reversals, strong $\mathrm{H} \beta$ excess emission and also detected the $\mathrm{Na}$ I $\mathrm{D}_{1}$ and $\mathrm{D}_{2}$ lines with broad wings. In that survey, the magnetic field indicator He I $\mathrm{D}_{3}$ was not detected, whereas Saar et al. (1997) observed the $\mathrm{He}_{\mathrm{I}} \mathrm{D}_{3}$ feature, again suggesting short-term variability.

Güdel (1992) observed V833 Tau with the VLA and measured a flux at $3.6 \mathrm{~cm}$ of $5.22 \pm 0.07 \mathrm{mJy}$ and computed a radio luminosity of $L_{\mathrm{R}}=0.2 \times 10^{15} \mathrm{ergs} \mathrm{s}^{-1} \mathrm{~Hz}^{-1}$. They also gave a value for the X-ray luminosity of $L_{\mathrm{X}}=0.8 \times$ $10^{29} \mathrm{ergs} \mathrm{s}^{-1}$. Later, Dempsey et al. (1997) measured $L_{\mathrm{X}}=$ $0.42 \times 10^{30} \mathrm{ergs} \mathrm{s}^{-1}$ and determined the following twocomponent coronal temperatures: $T_{\text {high }}=1.25 \times 10^{7} \mathrm{~K}$ 
and $T_{\text {low }}=2.52 \times 10^{6} \mathrm{~K}$. Tsikoudi \& Kellett (1997) found no variability of the star in the EUV 110-200 $\AA$ region with the ROSAT WFC, except for one possible flare.

Saar et al. (1990) determined the magnetic field strength of V833 Tau from a comparison of magnetically sensitive to insensitive absorption lines and obtained $B \approx 2600 \mathrm{G}$ with a filling factor of about $50 \%$. A similar result was found by Saar (1996) who then obtained $B \approx 2800 \mathrm{G}$ and a filling factor of about $60 \%$. Broadband linear polarization measurements by Saar et al. (1994) showed a rapid change in the amplitude and position angle of the polarization over a two hours period, that they interpreted as a result of flare-generated particle beams.

Recently, V833 Tau received special attention. Firstly, because it is a member of the Hyades cluster (Perryman et al. 1998) and thus has an evolutionary age of $625 \pm$ 50 Myrs and, secondly, the low mass secondary of the system $\left(f(m)=0.00022\right.$, Griffin et al. $1985 ; M_{\mathrm{sec}} \approx$ $0.05-0.17 M_{\odot}$, Halbwachs et al. 2000) appears on lists of brown dwarf candidates (see Cuntz et al. 2000; Halbwachs et al. 2000).

The long-term photometric behaviour of V833 Tau was investigated using photographic plates from 1899-1980 by Hartmann et al. (1981), and from 1898-1989 by Bondar (1995). The first photoelectric measurements were made in 1983 (see Oláh \& Pettersen 1991). Photoelectric monitoring of the star started in 1987 and has been carried out continuously since then. Strassmeier et al. (1997) summarized the photoelectric data from the literature and published new observations until 1996. V833 Tau was considered to have one of the longest starspot cycle of about 60 years based on the above mentioned photographic measurements. The next spot maximum (brightness minimum) was predicted for $\approx 1992$ by Saar et al. (1990), however, at that time the star was close to its previously measured maximum brightness and by 1998 it became even brighter. From a study of the 1987-1999 photometric data Oláh et al. (2000) found additional long-term cycles on the time scales of 6.4 and 2.5 years.

V833 Tau is one of the most active $\mathrm{K}$ dwarf stars exhibiting many interesting activity features and could be a Rosetta stone for the understanding of stellar cycles in very rapidly rotating stars. In this paper, we analyse all the available multicolour photoelectric data and investigate the activity of the star in time. The very low inclination of the star relative to the line of sight $\left(\approx 20^{\circ}\right)$ emphasizes the polar regions as the location of the observed activity. We also analyse two flares observed in 1983 and 1993, in $U B V R_{\mathrm{C}}$ and $U B V(R I)_{\mathrm{C}}$ colours, respectively.

\section{Observations}

For the present investigation the existing $V$ and $I_{\mathrm{C}}$ data of V833 Tau (until 1996 see Strassmeier et al. 1997) is supplemented with four more years of observations (19972000) made by the Amadeus $0.75 \mathrm{~m}$ automatic photoelectric telescope (T7-APT) of the University of Vienna at Fairborn Observatory in Arizona (Strassmeier et al. 1997).

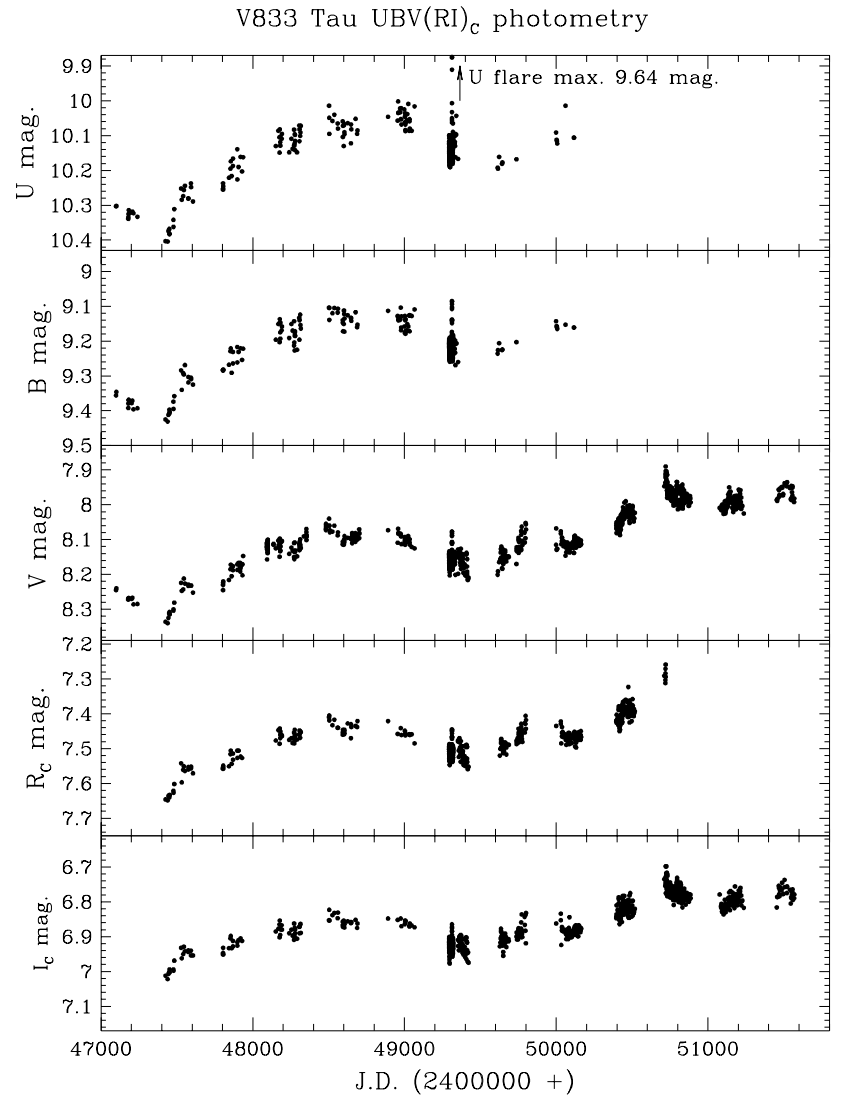

Fig. 1. New $U B V(R I)_{\mathrm{C}}$ observations of V833 Tau between 1987-2000.

We also present $U B V(R I)_{\mathrm{C}}$ observations made by the $0.8 \mathrm{~m}$ Automatic Photoelectric Telescope, then operated at Mt. Hopkins, Arizona, for Villanova University, as part of the Four College Consortium. These observations were obtained during late 1993 and included a huge flare visible in all five bandpasses. To match the Villanova observations with the data observed by the T7-APT and the observations from Catania (see Strassmeier et al. 1997), that were taken shortly after the Villanova observations, we applied corrections of $0{ }^{\mathrm{m}} 020,-0{ }^{\mathrm{m}} 010$ and $-0 .{ }^{\mathrm{m}} 015$ magnitudes in $V, R_{\mathrm{C}}$ and $I_{\mathrm{C}}$ colours, respectively, to the Villanova observations. The Hipparcos-Tycho data had to be shifted by $-0 .{ }^{\mathrm{m}} 122$ in $V$ to be in accord with other observations made during the same time. The magnitudes of the comparison star $\left(\mathrm{BD}+26^{\circ} 729\right)$ and the check star $\left(\mathrm{BD}+27^{\circ} 683\right)$ are given in Oláh \& Pettersen (1991). All the available photoelectric observations are presented in Fig. 1 and the colour indices in Fig. 2.

The four-colour data of the big flare observed on this star in 1983 (Oláh \& Pettersen 1991) were also analysed, thus its characteristics could be compared with the other flare presented in this paper.

To study the long-term behaviour of this star we digitized the photographic observations of Hartmann et al. (1981) from the published light curve. The long-term dataset is supplemented with the photographic measurements of Bondar (1995) and with the yearly mean 
V833 Tau colour indices

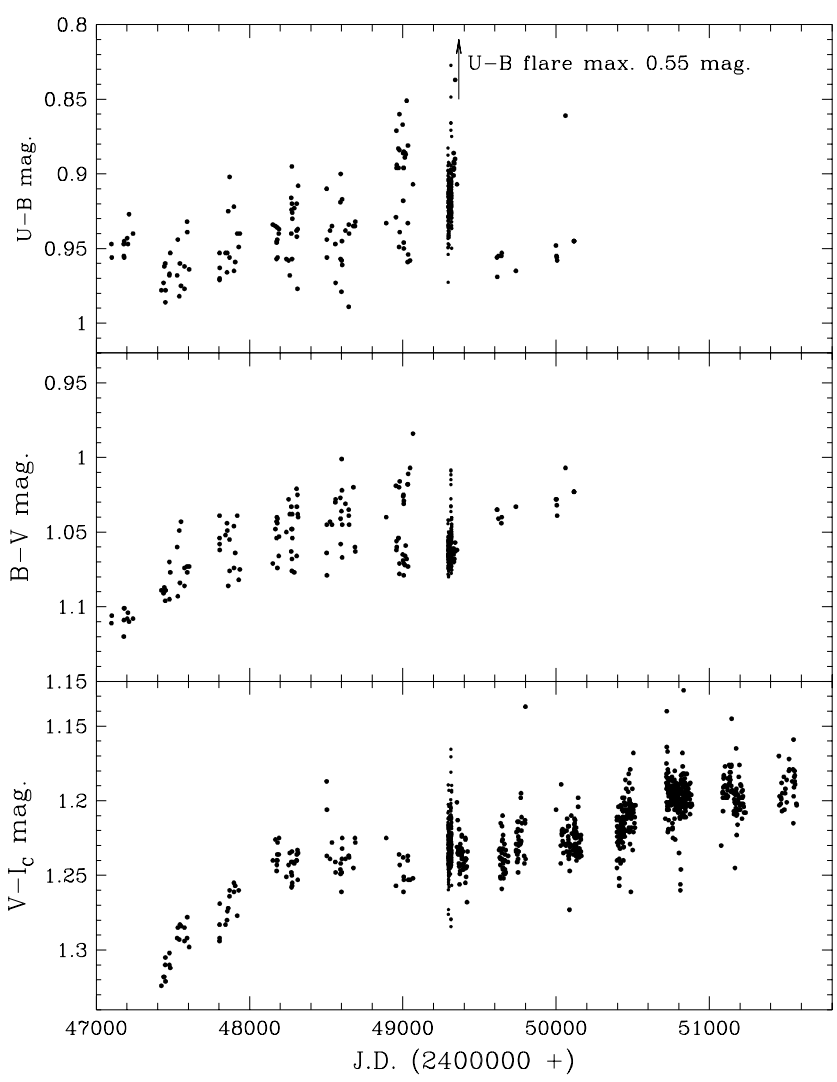

Fig. 2. Colour indices of V833 Tau between 1987-2000.

values of our photoelectric observations. Whenever available, we used the measured $B$ values. We add an average $B-V=1$. 063 to the Vienna APT $V$ data to get $B$ values; from the measured $B-V$ variability (see Fig. 2) we can estimate that the error of the resulting $B$ magnitudes is not higher than \pm 0 . 05 .

\section{Method}

For the period study, we used the program package MUFRAN (Kolláth 1990). For the unevenly sampled data it calculates discrete Fourier transforms and the recovered frequencies are refined by a non-linear least-square fits. Details and a test of this method are given in Oláh et al. (2000).

For the spot modeling, we assume that the light variations arise exclusively from dark (cool) starspots. As a first step, we selected those parts of the photometric data that were suitable for spot modeling. Because of the low inclination of the star $\left(\approx 20^{\circ}\right)$, the amplitude of the rotational modulation is small (seldom exceeding 0.1 ) and at several times the light variations are below the noise level. Altogether, 20 light curves in $V$ and $I_{\mathrm{C}}$ with sufficiently large rotational modulation above the noise level were found suitable for the analysis. For the starspot modeling and spot temperature determination we used our own computer code, based on Budding's (1977) equations, that assumes circular spot(s) which are

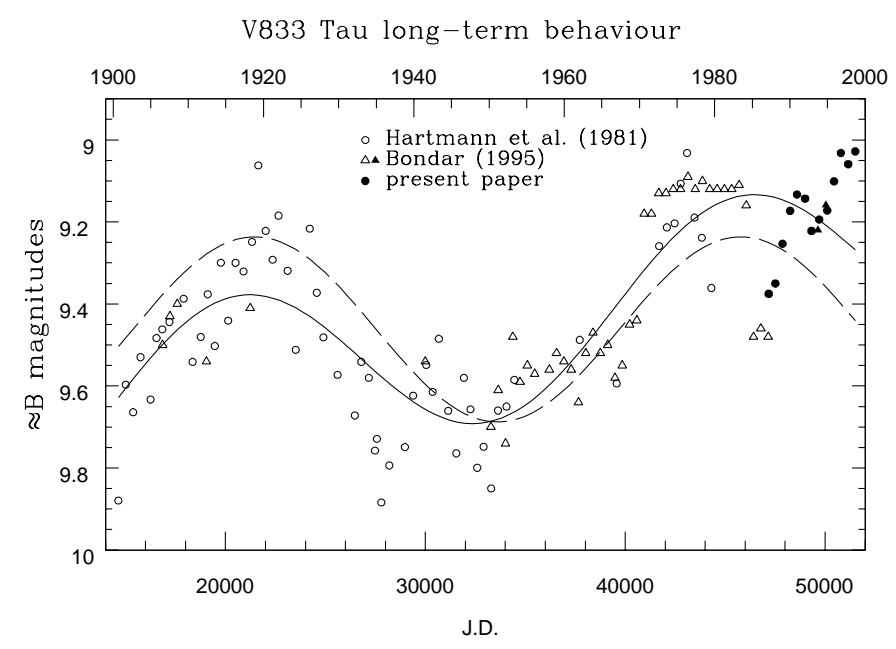

Fig. 3. Long-term blue light curve of V833 Tau during the last century. The dashed line represents a pure sinusoidal fit of about 67 years period, the solid line shows the fit with a linear trend plus a period of about 69 years.

homogenously cooler and thus darker than the surrounding photosphere. Only the spot's area and location (longitude and latitude) were adopted as free parameters. As a consequence of the low inclination, the light curves are nearly sinusoidal. Therefore, only one spot was needed to account for the variability. Two-spot fits were also performed, but the results were unstable, the six free parameters apparently overinterpreted the information content of the data.

For each dataset, we derived the spot temperature separately. We calculated the flux ratio between the unspotted surface and the spot using synthetic magnitudes from model atmospheres derived by Hauschildt et al. (1999). The unspotted stellar temperature was adopted to be $T_{\text {eff }}=4500 \mathrm{~K}$ based on fluxes from Houdashelt et al. (2000). For comparison, we generated a second set of models using the black body approximation. The same limb darkening values of 0.795 in $V$ and 0.572 in $I_{\mathrm{C}}$ were used for both the star and the spot (van Hamme 1993). With the help of Figs. 1 and 2 we adopted the maximum simultaneously observed magnitudes in $V=7^{\mathrm{m}} 90$ and in $I_{\mathrm{C}}=6.72$ as unspotted brightness at these wavelengths. The inclination of the star is low (i.e. nearly pole-on). Glebocki \& Stawikowski (1995) found $22^{\circ} \pm 6^{\circ}$; while Tokovinin (1990) found $11^{\circ} \leq i \leq 23^{\circ}$. For our modeling, we adopted an average value of $i=20^{\circ}$.

\section{Results and discussion}

\subsection{Multiple periodicity of V833 Tau}

Hartmann et al. (1981) and Bondar (1995) suggested a long-term cycle length of about 60 years, the longest of all known cycles of active stars. The collected data are presented in Fig. 3 together with fits of possible cycle lengths. A simple sinusoidal fit gives a cycle length of about 67 years. When a (linear) trend was also supposed, we found a similar value of 69 years. As seen in Fig. 3, the 
V833 Tau, $\mathrm{P}=2.39$ years
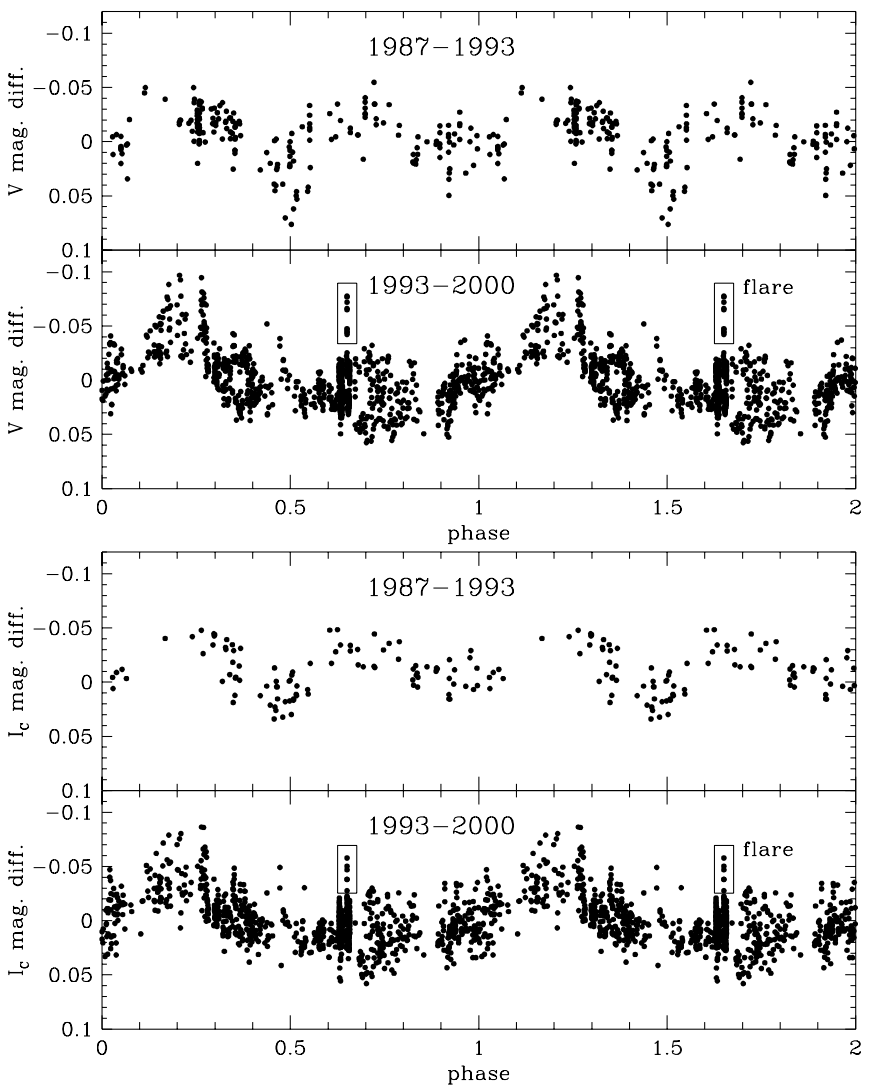

Fig. 4. The 2.39-years cycle of V833 Tau in $V$ (top) and in $I_{\mathrm{C}}$ (bottom). The 1993 flare event is marked.

length of the second cycle with a starting minimum during 1945-50, will be much longer than suspected because after 50 years the star is still at its maximum brightness.

We repeated the Fourier analysis from Oláh et al. (2000) for the now one-year-longer dataset, both in $V$ and $I_{\mathrm{C}}$ and find 6.7 years in $V$ and 6.3 years in $I_{\mathrm{C}}$ in good agreement with Oláh et al. (2000). After prewhitening the data with these periods plus a linear trend, the new result for the shorter cycle is 2.39 years in $V$ and 2.45 years in $I_{\mathrm{C}}$, the difference is not significant. The estimated error of the longer cycle length is about 0.7 years, and of the shorter one is about 0.1 years. The prewhitened data are folded with the 2.39 year cycle (found for the $V$ data) and these are displayed in Fig. 4.

There is no hope for identifying the few year long cycles in the long-term, less accurate photographic dataset. The amplitude of the longer cycle $(\approx 6.5$ years) is about $0 .{ }^{\mathrm{m}} 18$ and of the shorter one $(\approx 2.4$ years $)$ is $0 .{ }^{\mathrm{m}} 05-0 .{ }^{\mathrm{m}} 07$ while the scatter of the long-term photographic data in Fig. 3 is usually higher than 0.1 due to the (possible) rotational modulations and flares (see Fig. 1 of Bondar 1995). Moreover, the photographic observations are sparse, just one (or very occasion ally two) datapoints per annum.

The rotational period derived from the photometry is slightly longer than the orbital period, the latter recently determined by Halbwachs et al. (2000) to be
$P_{\text {orb }}=1.787992 \pm 0.000002$. Table 1 gives our results of the period determinations for three observing seasons where the rotational modulation was clearly detected during most of the time. The rotational period resulting from the study of the whole dataset is also given. The uncertainties of the periods are estimated on the assumption that the phases calculated with the given period should give a difference less than 0.2 at the extreme time intervals of the corresponding dataset (Walraven et al. 1992). The low inclination $\left(i \approx 20^{\circ}\right)$ of V833 Tau can be used to explain why we always observe longer rotational than orbital periods for the primary of a practically synchronised ( $e=0.002 \pm 0.005$, Halbwachs et al. 2000) binary. Assuming solar type differential rotation, i.e. the polar regions should rotate slower than the equator, the smaller rotational periods, reflected by the spots at high latitudes, dominate. Finally, we note again the similarities between V833 Tau and the Sun. Both stars exhibit three global variability timescales (apart from the rotation), that is 2.4, 6.4, and 50-80 years for V833 Tau and 11, 80-90 (Gleissberg 1967), and 200-300 (cf. the Introduction of Juckett 2000, and references therein) years for the Sun.

Table 1. Rotational periods from photometry.

\begin{tabular}{cccc}
\hline Year & $\begin{array}{c}\text { period } \\
\text { (days) }\end{array}$ & $\begin{array}{c}\text { time span } \\
\text { (days) }\end{array}$ & $\begin{array}{c}\text { number of } \\
\text { observations }\end{array}$ \\
\hline $1993-1994$ & $1.7996 \pm 0.0022$ & 127 & 278 \\
$1996-1997$ & $1.8047 \pm 0.0049$ & 122 & 135 \\
$1997-1998$ & $1.7889 \pm 0.0027$ & 174 & 234 \\
\hline $1987-2000$ & $1.7940 \pm 0.0005$ & 4472 & 1143 \\
\hline$P_{\text {orb }}$ & $1.787992 \pm 0.000002$ & & \\
\hline
\end{tabular}

\subsection{Light curve modeling}

The initial step for modeling the light curves was to derive spot temperatures for each of the 20 datasets. The $V-$ $I_{\mathrm{C}}$ color index curves were modeled using different spot temperatures between $\Delta T=100-2000 \mathrm{~K}\left(\Delta T \equiv T_{\text {star }}-\right.$ $\left.T_{\text {spot }}\right)$. The $\Delta T$ value at the minimum of $\chi^{2}$ (goodness of fit) for each dataset is adopted as the most likely spot temperature. The 20 light and colour curves and their fits are displayed in Fig. 5, the corresponding spot sizes and configurations are plotted in Fig. 6.

The very low inclination of the star, together with the large long-term light variability that reached $\approx 0.7$ during the last century, argues strongly in favour of high-latitude spots. This is very similar to the case of BY Dra, where a long-term variability of 0.6 , and a low inclination of $\approx 30^{\circ}$, obviously suggest polar spottedness on one or both K-dwarf components of this binary (cf. Kővári 1999).

Our spot models are not mathematically unique solutions to the light curves (cf. Kővári \& Bartus 1997) but represent the simplest possible solutions for the data between 1990-2000. This time range covers a 0.25 change of the mean brightness together with the comparably 
K. Oláh et al.: Time-series photometric spot modeling. IV.
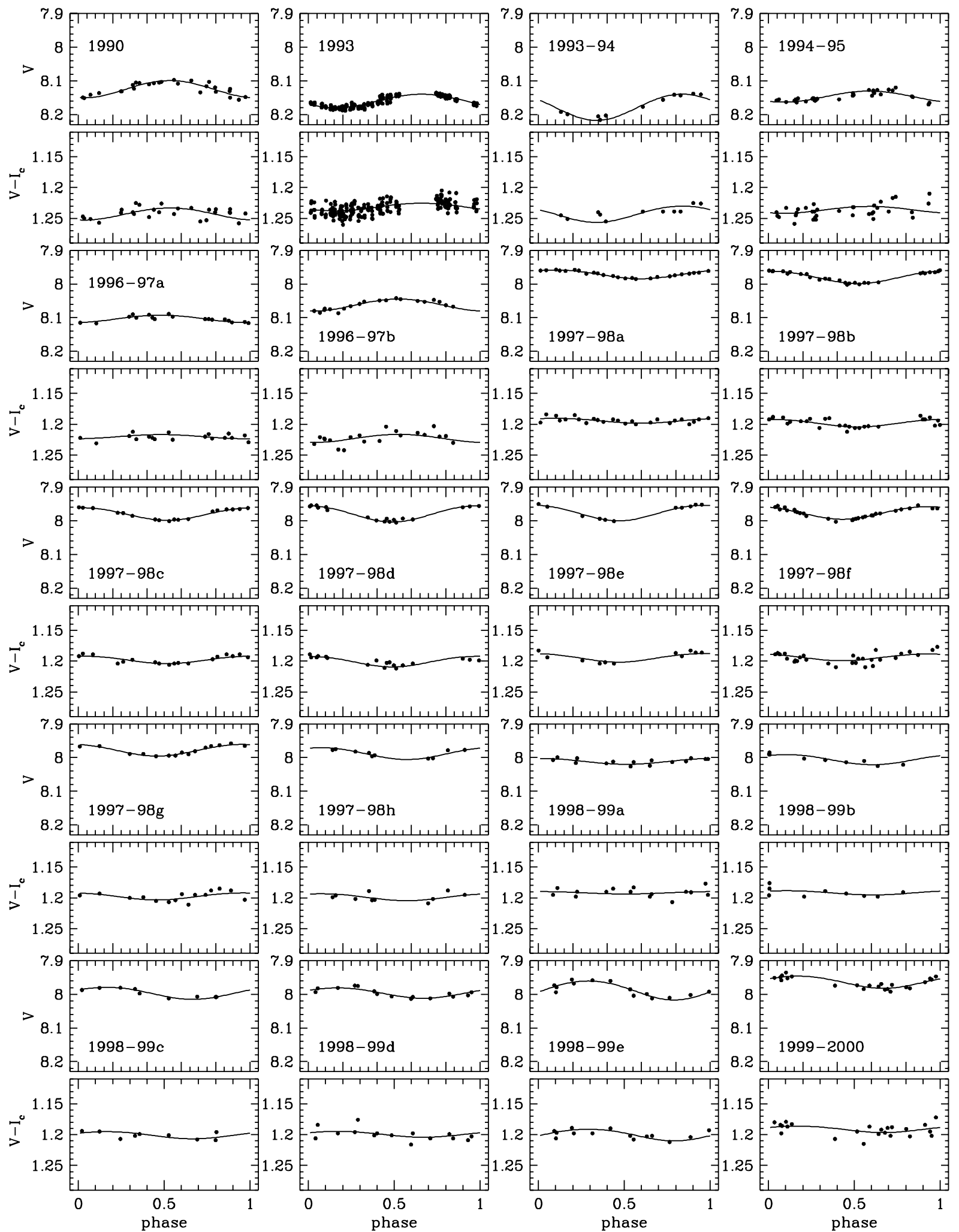

Fig. 5. Fitted $V$ band and $V-I_{\mathrm{C}}$ colour index curves on the same scales. The upper line of each $V$ box mark the accepted unspotted brightness of the star $(V=7.90 \mathrm{mag})$.

low-amplitude rotational modulation. The large amplitude of the long-term brightness changes suggests the existence of big spots during low system brightness that should be visible at least partly all the time, i.e. well over $20^{\circ}$ stellar latitude, and account for both the rotational modulation and the long-term variability. It is likely, that the spot configuration of V833 Tau is more complex than we depict. Because of its poleward orientation, our 

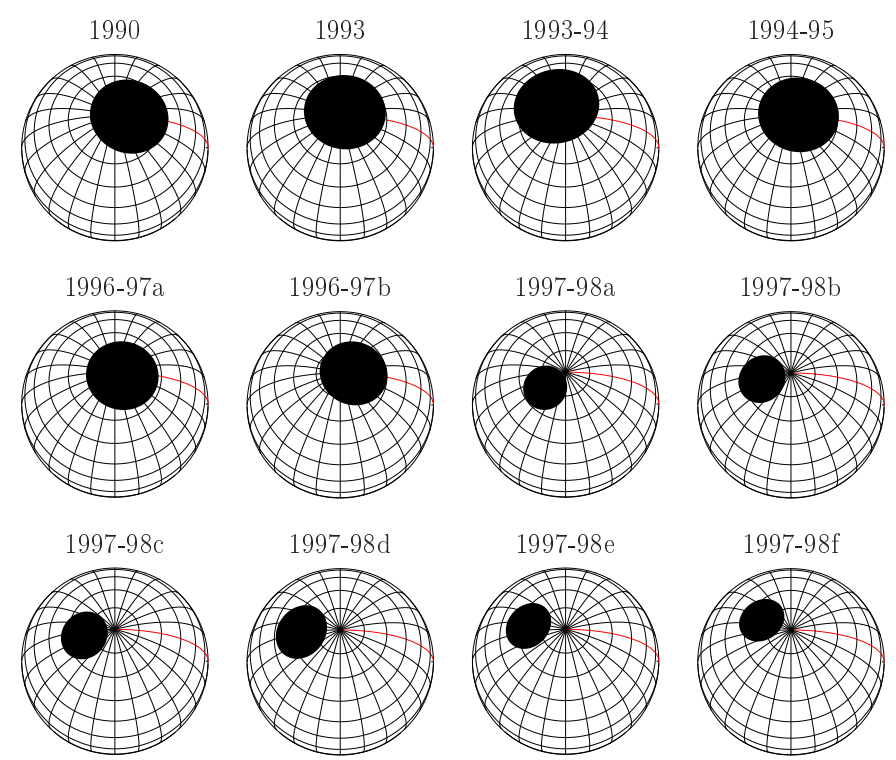

$1997-98 \mathrm{~h}$
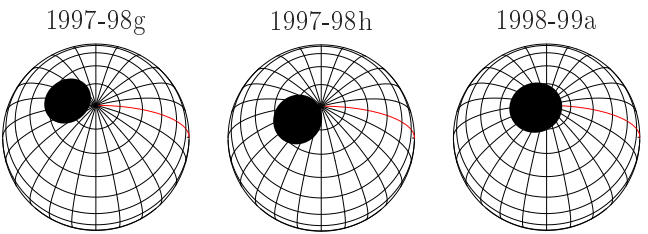

1998-99d
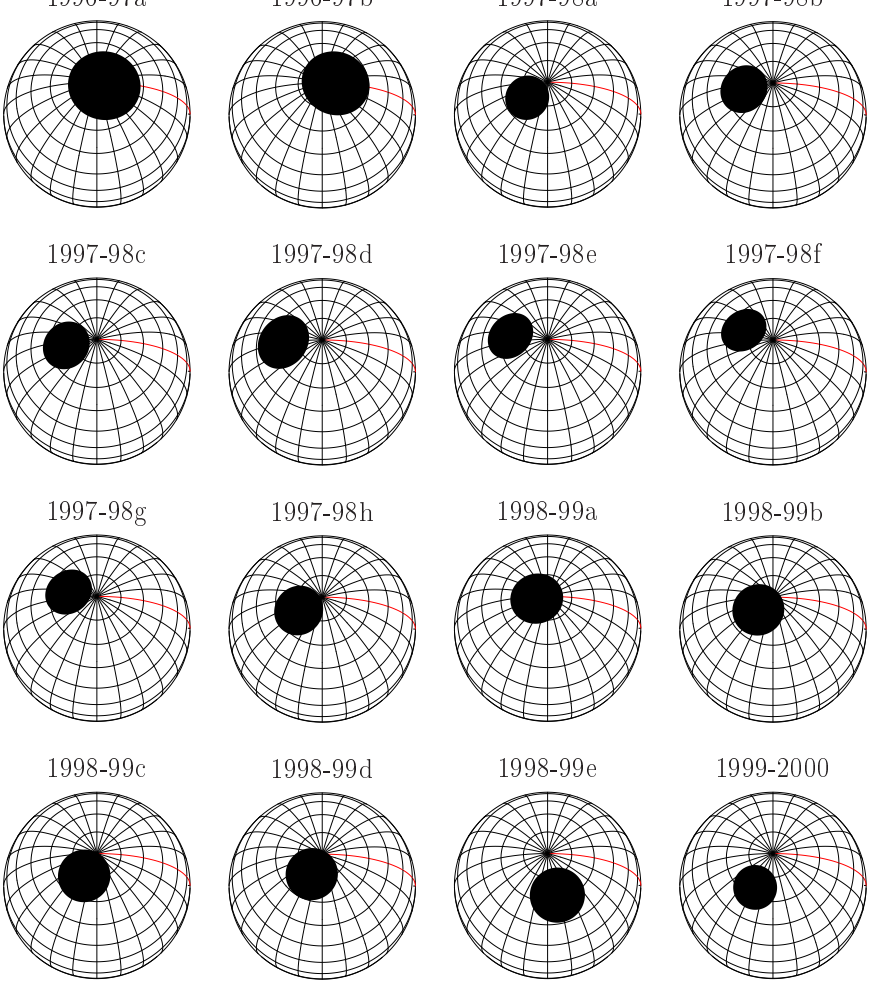

1997-98f

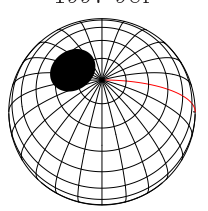

$1998-99 b$

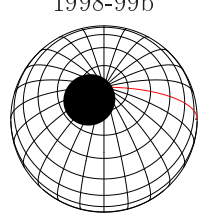

$1999-2000$

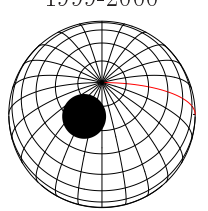

Fig. 6. One-spot model for each photometric dataset in Fig. 5.

modeling is most sensitive to spots located at high stellar latitudes, and is insensitive to spots that could be located near the equator.

However, the presence of polar or at least very high latitude spots is very likely on low-mass, rapidly rotating stars. The latitude emergence pattern of flux tubes on low-mass stars has recently been modeled by Granzer et al. (2000). If we adopt a mass of $\approx 0.8 M_{\odot}$ (Strassmeier et al. 1993) and $\Omega / \Omega_{\odot} \approx 15$ for V833 Tau then, according to Granzer et al.'s results, spots on V833 Tau should reach the surface within stellar latitudes of $25^{\circ}$ to $70^{\circ}$. Our average spot latitude is slightly higher than $70^{\circ}$, but this still agrees fairly well with the models, especially if we take into account that the model of Granzer et al. (2000) gives only the approximate emergence latitude from where the spots can drift even higher. Low latitude features, comparable to those given by the models, were found when we used the two-spot approximation, but because of the very low amplitude of the light variation, those solutions were not stable.

\subsection{Spot temperature and area changes}

The spot temperatures and areas from the 20 modeled light curves are listed in Table 2 . The uncertainties of the temperature values are typically $150-200 \mathrm{~K}$. As a test, we assumed an unspotted brightness brighter by 0.2 in $V$ than originally obtained, i.e. increased from $V=7^{\mathrm{m}}$.90 to $V=7^{\mathrm{m}} \cdot 70$. From the $V-I_{\mathrm{C}}$ color index curve it is seen that the star is bluer by 0.03 when it brightens by 0.2 . Thus $I_{\mathrm{C}}$ should be increased by 0.17 (from $I_{\mathrm{C}}=6^{\mathrm{m}} \cdot 72$ to $I_{\mathrm{C}}=$ $6.55)$ to match the color index at the higher assumed unspotted light. With these artifically higher unspotted values we redid the modeling.

When we adopted the observed maximum as the unspotted brightness, the average spot temperature we found was $\overline{\Delta T}=1230 \mathrm{~K} \pm 270 \mathrm{~K}$. On the other hand, we obtained $\overline{\Delta T}=1480 \mathrm{~K} \pm 100 \mathrm{~K}$ when using a 0.2 brighter unspotted brightness than observed. The difference of $260 \mathrm{~K}$ is probably not resolvable from our photometry. The resulting individual spot temperatures and areas together with the $V$ light curve are plotted in Fig. 7 .

These temperatures are in good agreement with the result of Saar et al. (2000) who determined $\Delta T=1330 \mathrm{~K} \pm$ $110 \mathrm{~K}$ by modeling TiO bands. The stellar (photospheric) temperature they derived was $T_{\text {eff }}=4510 \mathrm{~K} \pm 110 \mathrm{~K}$. This value is practically identical to the one we used $\left(T_{\text {eff }}=\right.$ $4500 \mathrm{~K}$ ) in the modeling, which was based on the tables of Houdashelt et al. (2000) using the measured colour indices of the star at its brightest observed state.

Saar et al. (2000) found a spot filling factor of about $50 \%$, based on observations taken between 1989-1994. However, our highest spot coverage is found in 1994, when it was $5.6 \%$ of the total stellar surface; that corresponds to $11.2 \%$ of half of the stellar surface. Saar et al.'s (2000) $50 \%$ filling factor could represent an about $30 \%$ large circular spot at the disc center (for explanation see Fig. 8 of O'Neal et al. 1996), but this is still about three times larger than our spot area estimate. Using higher unspotted brightness by 0.2 than the observed maximum, our spot area increased by $1.5-2.5$ times, reaching $17.8 \%$ of half of the surface in 1994, but that is still less than $2 / 3$ of the spot area found by of Saar et al. (2000). The agreement is not very good between the two spot filling factor results using different methods, but are not inconsistent with each other.

The cause of the discrepancy is unknown at present. Saar et al.'s (2000) observations were taken between 19891994, when the star was about 0.2 fainter than the historical maximum. If the spot coverage is about $50 \%$ at the stellar brightness of 0.2 below the historical maximum (which is, in fact, the faintest possible unspotted brightness), a filling factor at the time of the historical minimum between 1945-1950, when the star was an additional $0 .{ }^{\mathrm{m}} 6$ fainter than between 1989-1994, would be even larger.

On the other hand, for $\sigma$ Gem O'Neal et al. (1998) found that a filling factor derived from $\mathrm{TiO}$ bands matched well the photometric spot coverage. The observed (by Strassmeier et al. 1993) and computed (O'Neal et al. 1998) 
Table 2. Spot temperatures and areas using either synthetic colours or a blackbody approximation.

\begin{tabular}{|c|rc|r|r|rc|}
\hline & \multicolumn{6}{|c|}{ unspotted brightness } \\
\hline & \multicolumn{5}{|c|}{ obs.max } & \multicolumn{2}{c|}{ obs.max+0.2 } \\
\hline mean & \multicolumn{2}{|c|}{ synth. col. } & \multicolumn{2}{|c|}{ BB approx. } & \multicolumn{2}{|c|}{ synth. col. } \\
JD & $\Delta T^{a}$ & $\left(\%^{d}\right)$ & \multicolumn{1}{|c|}{$\Delta T^{b}$} & $\left(\%^{d}\right)$ & $\Delta T^{c}$ & $\left(\%^{d}\right)$ \\
\hline \hline 48242 & 950 & 4.8 & 650 & 7.5 & 1300 & 8.1 \\
49306 & 1300 & 5.1 & 1200 & 5.5 & 1500 & 8.4 \\
49411 & 1200 & 5.6 & 1050 & 6.6 & 1400 & 8.9 \\
49649 & 1150 & 4.9 & 950 & 5.7 & 1400 & 8.3 \\
50121 & 1300 & 4.0 & 1200 & 4.3 & 1500 & 7.4 \\
50413 & 1000 & 3.5 & 750 & 4.8 & 1300 & 7.0 \\
50754 & 1250 & 1.5 & 1150 & 1.7 & 1500 & 5.1 \\
50777 & 1100 & 1.8 & 900 & 2.2 & 1500 & 5.3 \\
50784 & 1100 & 1.8 & 900 & 2.2 & 1500 & 5.2 \\
50804 & 900 & 2.0 & 550 & 3.1 & 1400 & 5.4 \\
50816 & 1250 & 1.8 & 1050 & 2.0 & 1500 & 5.2 \\
50832 & 1300 & 1.7 & 1150 & 1.8 & 1500 & 5.2 \\
50843 & 1150 & 1.8 & 900 & 2.1 & 1500 & 5.2 \\
50877 & 1150 & 1.9 & 1000 & 2.2 & 1500 & 5.4 \\
51095 & 2000 & 2.1 & 2000 & 2.1 & 1700 & 5.7 \\
51120 & 1850 & 2.1 & 1900 & 2.1 & 1700 & 5.6 \\
51163 & 1150 & 2.1 & 950 & 2.4 & 1500 & 5.6 \\
51190 & 1250 & 2.0 & 1050 & 2.2 & 1500 & 5.6 \\
51214 & 1050 & 2.3 & 800 & 2.8 & 1400 & 5.6 \\
51517 & 1300 & 1.5 & 1200 & 1.6 & 1500 & 5.0 \\
\hline
\end{tabular}

${ }^{a}$ Average $\Delta T=1230 \mathrm{~K}$.

${ }^{b}$ Average $\Delta T=1070 \mathrm{~K}$.

${ }^{c}$ Average $\Delta T=1480 \mathrm{~K}$.

${ }^{d}$ In percent of the total stellar surface. If we assume a northsouth symmetric polar spot configuration, then the spotted area of the star is doubled (we never see the other polar region).

"unspotted" $V$ magnitude agreed. This fact shows that both methods can agree and work properly. About spot coverage, using the Sun as a proxy, we showed that if the unspotted brightness level and the flux ratio between the spot and the undisturbed surface is well known, then the resulted spot area matches well the spot area derived from direct images (for more details see Oláh et al. 1999).

From our continuous observations of V833 Tau in Fig. 7 we find some indications of spot temperature changes. When the spot temperature relative to the photosphere $\left(\Delta T=T_{\text {star }}-T_{\text {spot }}\right)$ is smaller (warmer spots), there may be some marginal evidence that the spot area is also larger (Fig. 7, lower panels). We suppose that when a new spot (or a group of spots) appears on the star, it is cooler relative to the photosphere $(\Delta T \approx 1500-2000 \mathrm{~K})$. The spot appearence is followed by a longer time interval, when the newly emerged magnetic structure around and in the spot relaxes. The spot temperature seems to approach an asymptotic value, which is about 900-1000 K cooler relative to the photosphere in case we use the historical maximum as the unspotted brightness. We get similar results using a higher unspotted brightness, although the spot temperatures are lower and vary less. There is just a few pieces of evidence about variable spot temperatures in the literature. Amado et al. (2000) found changing spot
V833 Tau spot temperature and area 1990-2000

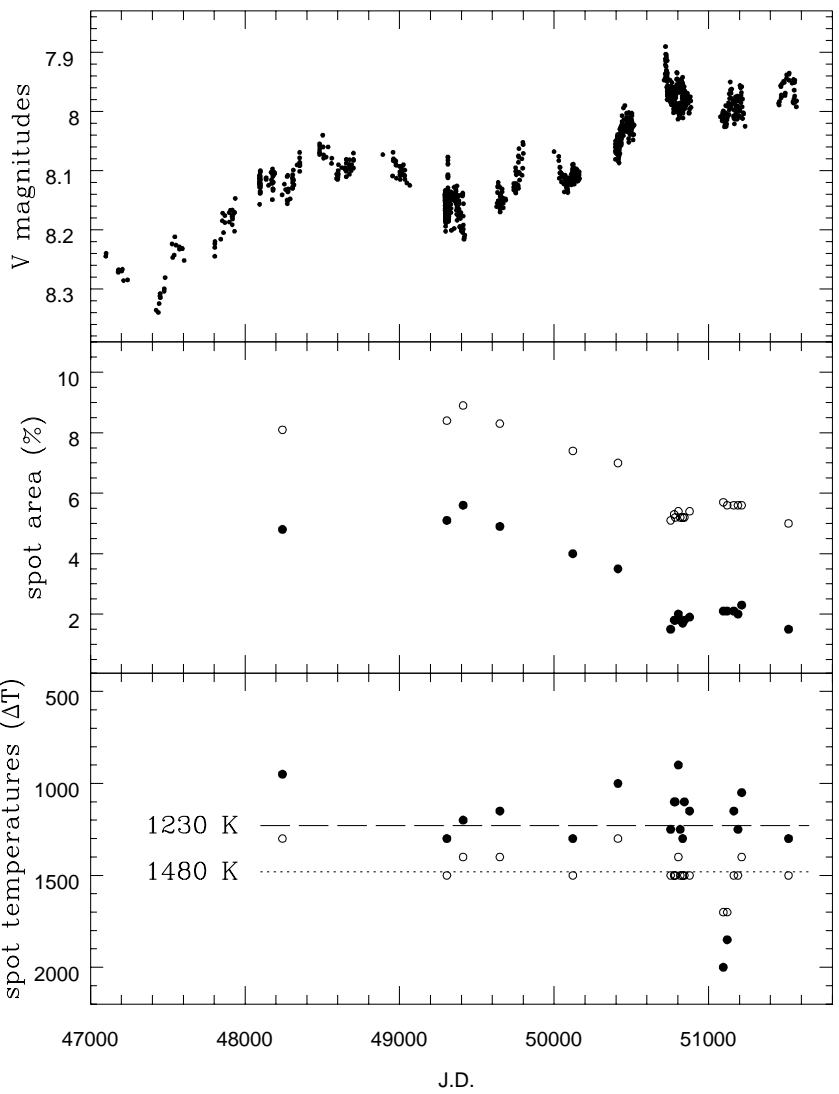

Fig. 7. $V$ observations (top), spot area change (middle) and spot temperature change (bottom) of V833 Tau during the last decade. Dots mark the results when using the observed maximum as unspotted brightness, circles denote the results when the unspotted light was assumed to be higher than the observed maximum by 0.2 in $V$. See text.

temperatures on AB Dor between 1987-1997 from multicolour photometry. Spots with different temperatures were found on II Peg by Byrne et al. (1995) in 1991, also from multicolour photometry and again in 1996 from spectroscopic modeling of TiO bands by O'Neal et al. (1998).

If these spot temperature changes are indeed real, they could in part be due to contributions from varying filling factors of white light faculae and/or plages. The plages appear in the chromosphere above facular areas as heated regions, representing much less energy output compared with the underlying faculae. A change in the spot temperature of V833 Tau could be explained through a solar analogue in that its spots decay faster than its plages (e.g. Howard 1992). On V833 Tau, where we presume we are observing decaying spot(s), the relative area of plages to spots could be increasing. It would be reasonable to presume that the ratio of the underlying faculae to spots could be increasing in the same way. Since we model the active region as a homogeneous area, we obtain an increasing spot temperature with time. 


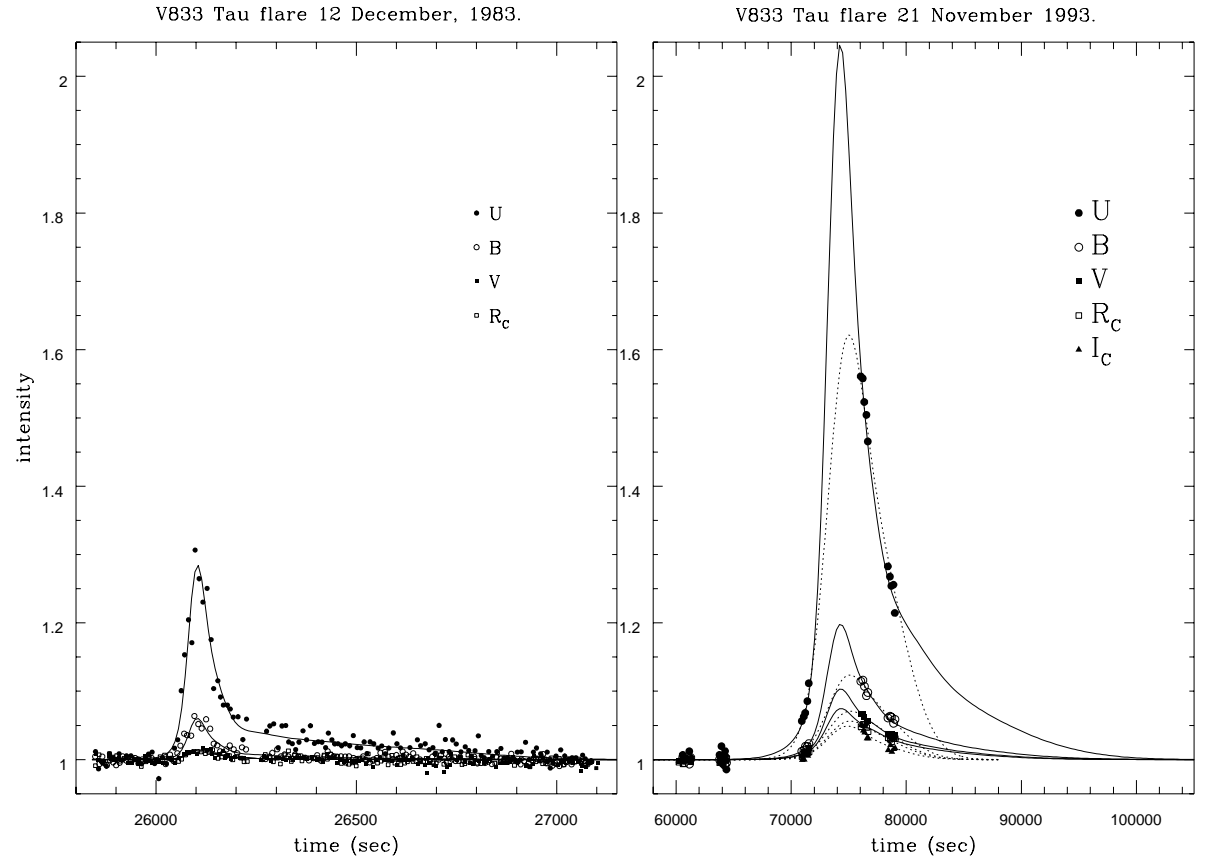

Fig. 8. Observations and fits for the 12 Dec. 1983 flare (left) and the 21 Nov. 1993 flare (right) light curves in $U, B$, $V, R_{\mathrm{C}}$, and $I_{\mathrm{C}}$ (from top to bottom, respectively). The dashed line in the right panel corresponds to the "minimum fit", see text.

\section{The 12 Dec. 1983 and the 21 Nov. 1993 flares}

In this section, we briefly discuss two flare events that occured on 12 Dec. 1983 and 21 Nov. 1993 UT. The data of the 1983 flare were obtained in the $U B V R_{\mathrm{C}}$ bands at McDonald Observatory, for details see Oláh \& Pettersen (1991). The 1993 flare was observed in the $U B V(R I)_{\mathrm{C}}$ bands with the $0.8 \mathrm{~m}$ Villanova APT.

In both cases, towards longer wavelengths the flare peak intensity lies significantly above the quiescent radiation level of the star. During the flares the $U-B$ colour index showed excess of few tenths of magnitudes, suggesting as high as $\approx 10^{4} \mathrm{~K}$ colour temperature for the flaring area. However, the two flares occured on different time scales and energetic ranges. The 12 Dec. 1983 flare was shorter $\left(\Delta t \approx 10^{3} \mathrm{~s}\right)$ and less energetic than the flare observed on 21 Nov. $1993\left(\Delta t \approx 10^{4} \mathrm{~s}\right)$, this latter is a typical time scale for solar white light two-ribbon flares (Haisch 1989).

Using the observations obtained in five colours on JD 2449312.70, i.e. before the flaring phase of the 21 Nov. 1993 event, we determined the flux originated from the quiescent stellar surface. For this we adopted the surface flux values from the Buser \& Kurucz (1992) tables assuming solar abundance with $T_{\text {eff }}=4500 \mathrm{~K}, \log g=4.5$, and taking $R_{*}=0.8 R_{\odot}$ (Naftilan \& Fairchild 1993). The second column of Table 3 gives the total quiescent stellar fluxes $\left(F_{\mathrm{S}}\right)$ in ergs/s, i.e.,

$F_{\mathrm{S}}=\int_{\lambda_{1}}^{\lambda_{2}} S(\lambda) * T_{\lambda_{1}-\lambda_{2}}(\lambda) \mathrm{d} \lambda$

for the different photometric filters between $\lambda_{1}$ and $\lambda_{2}$, where $S(\lambda)$ is the power function and $T_{\lambda_{1}-\lambda_{2}}(\lambda)$ is the transmission function for a given photometric filter.

We estimate the energy release by the flares in the optical wavelengths by fitting the flare light curves. The
1983 data were obtained only in four bandpasses and have lower quality than the 1993 observations. On the other hand, the light curve of the 21 Nov. 1993 flare is poorly covered, not permitting the full exploitation of the 1993 data. Therefore, for the 1993 observations two probable flare-curve shapes are assumed, one with an abrupt rising phase and a gently sloped cooling phase, copying the shape of the 1983 curves, while the other one is a "minimum fit". In the following we refer to the latter fit as values in parentheses. The fitted flare light curves are displayed in Fig. 8. For comparison, in Fig. 9 the $U$ fits are plotted on the same scale. The flare duration time was estimated upon the $U$ fit and found to be $t_{U} \approx 1200 \mathrm{~s}$ for the 12 Dec. 1983 flare and 27500 (15300)s for the flare on 21 Nov. 1993. The integrated net flare energy and the flare luminosity at maximum is given in Table 3 for the different photometric bandpasses.

Applying the method detailed in de Jager et al. (1986), we give an estimation for the colour temperature of the two flares at their maximum. The flux ratio between the flaring and the quiescent star is

$\left[F_{\mathrm{S}}(1-A)+F_{\mathrm{F}} A\right] / F_{\mathrm{S}}=1-A+A B_{\mathrm{F}} / B_{\mathrm{S}}$

where $F_{\mathrm{S}}$ is the total flux of the quiescent star (Table 3 ), $F_{\mathrm{F}}$ is the flare flux, $A$ is the ratio between the projected area of the flaring region and the visible stellar surface, $B_{\mathrm{F}}$ and $B_{\mathrm{S}}$ are the blackbody (BB) fluxes of the flare and the star, respectively. Note that there are two unknown quantities in Eq. (2), $A$ and $T_{\mathrm{F}}$, the flare temperature included in $B_{\mathrm{F}}$. By trial-and-error, we find solutions for a wide range of the coupled parameters (see Table 4).

In Fig. 10 the flare luminosity at maximum is plotted vs. effective wavelength. Also plotted are the most appropriate BB models (see also as boldface pairs in Table 4). We would expect the energy distributions of 
Table 3. Calculated luminosities for the quiescent star, and the total energy and luminosity at flare maximum for both flares in the five photometric bands. Values in parenthesis resulted from the "minimum fit".

\begin{tabular}{|c|c|c|c|c|c|}
\hline $\begin{array}{c}\text { colour } \\
\text { with } \lambda_{\text {eff }} \\
{[\AA]}\end{array}$ & $\begin{array}{c}\text { quiescent } \\
\text { radiation } \\
10^{31}[\mathrm{ergs} / \mathrm{s}]\end{array}$ & $\begin{array}{c}12 \text { DEC } \\
\text { energy } \\
10^{32} \text { [ergs] }\end{array}$ & $\begin{array}{l}1983 \text { FLARE } \\
\text { max luminosity } \\
10^{27}[\mathrm{ergs} / \mathrm{s} / \AA]\end{array}$ & $\begin{array}{c}21 \mathrm{NOT} \\
\text { energy } \\
10^{32} \text { [ergs] }\end{array}$ & $\begin{array}{l}1993 \text { FLARE } \\
\text { max luminosity } \\
\left.10^{27} \text { [ergs/s/ }\right]\end{array}$ \\
\hline$U-3660$ & 0.25 & 0.95 & 1.08 & $163(91)$ & $4.67(2.33)$ \\
\hline$B-4380$ & 1.37 & 0.90 & 0.89 & $156(105)$ & $2.88(1.82)$ \\
\hline$V-5450$ & 1.93 & 0.30 & 0.31 & $125(86)$ & $2.27(1.58)$ \\
\hline$R_{\mathrm{C}}-6410$ & 4.08 & 0.56 & 0.33 & $206(147)$ & $2.21(1.71)$ \\
\hline$I_{\mathrm{C}}-7980$ & 2.96 & - & - & $98(79)$ & $1.05(0.94)$ \\
\hline
\end{tabular}

Table 4. Values for flare temperature $T_{\mathrm{F}}$ and relative area $A=A_{\mathrm{F}} / A_{*}$ as a result of our trial-and-error procedure (see text). Best fit values are in boldface.

\begin{tabular}{|c|c|c|c|c|c|c|c|}
\hline \multicolumn{8}{|c|}{12 DEC. 1983 FLARE } \\
\hline$T_{\mathrm{F}}$ & 8000 & 8500 & 9000 & 10000 & 12000 & 15000 & 20000 \\
\hline$A$ & - & - & 0.0047 & 0.0031 & 0.0016 & 0.0008 & 0.0004 \\
\hline \multicolumn{8}{|c|}{21 NOV. 1993 FLARE } \\
\hline$\overline{T_{\mathrm{F}}}$ & 8000 & 8500 & 9000 & 10000 & 12000 & 15000 & 20000 \\
\hline$A$ & - & 0.0250 & 0.0200 & 0.0125 & 0.0065 & 0.0035 & 0.0017 \\
\hline & \multicolumn{7}{|c|}{ minimum fit: } \\
\hline$T_{\mathrm{F}}$ & 8000 & 8500 & 9000 & 10000 & 12000 & 15000 & 20000 \\
\hline$A$ & 0.0175 & 0.0135 & 0.0100 & 0.0070 & 0.0035 & 0.0017 & - \\
\hline
\end{tabular}

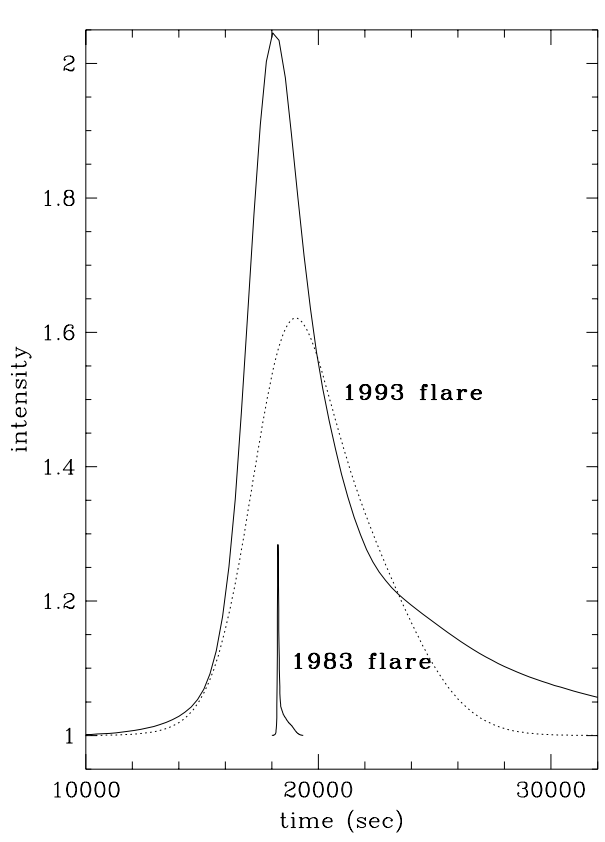

Fig. 9. Comparison of the energy and the time scales of the two flares. Plotted are the $U$ fits on the same time and flux scales. The dashed line corresponds to the minimum fit of the 1993 flare light curve.

the two flares to have a peak somewhere around $3000 \AA$ (cf., Pettersen 1988). However, it is seen that in 1993, towards the red wavelengths, there is a notable rise. Our solutions were confined to finding BB fits for the $U, B$ and $V$ colours only (above $T_{\mathrm{F}}=15000 \mathrm{~K}$ Planck curves do not fit even $V$ ). This is supported by the ratio of the 1993 to 1983 flare energy values listed in Table 3. For

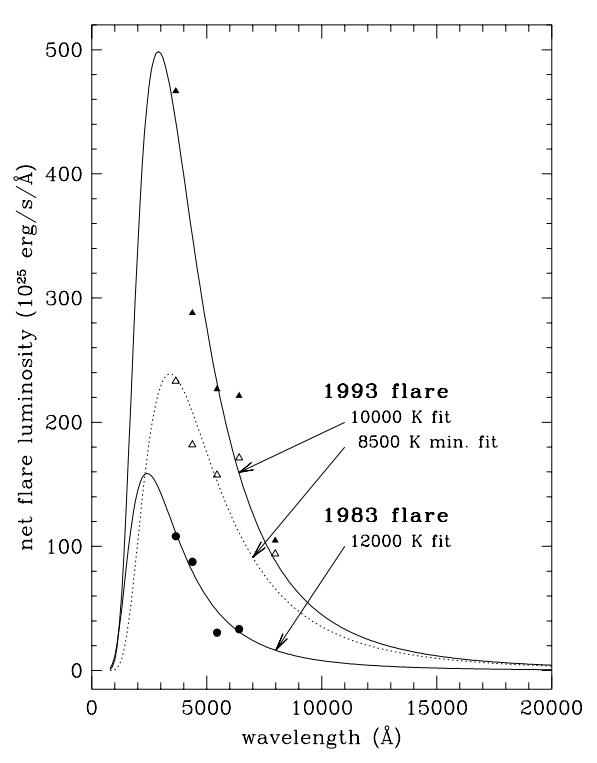

Fig. 10. The observed net flare luminosity density at the effective wavelength of the corresponding photometric bands for the flares on 12 Dec. 1983 (dots) and 21 Nov. 1993 (filled and empty triangles). Appropriate BB models are also shown (lines). The $R_{\mathrm{C}}$ excess in 1993 could not be fitted with a Planckian energy distribution.

the ratio $E_{C_{i}}^{1993} / E_{C_{i}}^{1983}$ we get $2-3$ times higher values for $C_{i}=V$ and $R_{\mathrm{C}}$ than for $C_{i}=U$ and $B$. The excess red flux of the 21 Nov. 1993 flare can also be shown statistically. According to our estimation in Table 3, the energy of the flare that is released in the optical bands is $E_{\mathrm{opt}}=E_{U}+E_{B}+E_{V}+E_{R_{\mathrm{C}}}+E_{I_{\mathrm{C}}}=7.47(5.09) \times$ $10^{34} \mathrm{erg}$, with $E_{\mathrm{opt}} / E_{U}=4.6(5.6)$, which is more than twice as much as the statistical value of $\approx 2.1$ given by 
Lacy et al. (1989) for dwarf flare stars, and is close to the upper limit of $\approx 5$ that Pettersen (1989) found for this ratio. Additionally, the sum of the energy emitted in $V, R_{\mathrm{C}}$, and $I_{\mathrm{C}}$ is $57(61) \%$ of the total optical energy of the flare, while Doyle et al. (1989) generally argued for half of this value, $E_{V R I} / E_{\mathrm{opt}}=1 / 3$. To account for this excess, nonthermal processes need to be considered. However, the time coverage of the 1993 data, and the lack of simultaneous spectroscopic observations, particularly in the $U V$, do not support further exploration of this problem. Nevertheless, such excess of the continuum at longer wavelengths is similar to the solar type-I white light flares (Fang \& Ding 1995), where the Balmer photons produce overheating in the upper photosphere (Machado et al. 1989).

Saar et al. (1994) obtained broad-band linearpolarization measurements of V833 Tau in 1988, and observed a significant change in both the magnitude and the angle of the polarization that lasted for about 2.6 hours. They concluded that particle beams generated by a large flare is the most plausible interpretation. The total flare energy was estimated to be about $10^{33}-10^{34}$ when a flare area coverage of $\approx 0.005$ was assumed. This flare thus should have been similar in duration and energy than the powerful 1993 flare observed by us.

\section{Summary}

- The combined photographic and photoelectric $B$ measurements of V833 Tau show a cyclic behaviour on a timescale of 70 years or longer, with an amplitude of about $0{ }^{\mathrm{m}} 6$. Using only the photoelectric observations between 1987-2000, variability on two shorter time scales of $\approx 6.5$ and $\approx 2.4$ years with amplitudes of 0.18 and $0 .{ }^{\mathrm{m}} 05-0{ }^{\mathrm{m}} 07$, respectively, is confirmed;

- The spot-area change generally follows the brightness variation of the star. The individual spot temperature remains fairly constant and gives an average spot temperature of $\overline{\Delta T}=1230 \mathrm{~K} \pm 270 \mathrm{~K}$ (rms) using the historical $V$ maximum as the unspotted brightness. An arbitrarily higher unspotted brightness by 0.2 results in $\overline{\Delta T}=1480 \mathrm{~K} \pm 100 \mathrm{~K}$ (rms). During 1997-1999, we detect short-term spot temperature and area changes that possibly could be active-region evolution. The relative area of faculae/plages to spots increases as the spots decay, therefore, we observe a net increase of the spot temperature;

- A powerful flare was observed in all five $U B V(R I)_{\mathrm{C}}$ bands in 1993 that lasted for about 4 hours. We analysed this flare and compared its characteristics to a less intense flare observed in 1983 (Oláh \& Pettersen 1991). The 1993 flare released $5-7 \times 10^{34} \mathrm{ergs}$ in the optical bands, $\approx 60 \%$ of this energy was emitted in the $V(R I)_{\mathrm{C}}$ bands. Such a red excess is reminiscent of the solar white-light flare energy distribution. The other flare in 1983 emitted less than $10^{32}$ ergs. For the 1993 and 1983 events, the flare temperatures are 8500-10000 K and $12000 \mathrm{~K}$, respectively, and the flare areas are about $1.3 \%$ and $0.2 \%$ of the stellar surface, respectively.

Acknowledgements. The authors are grateful to S. Saar and to L. van Driel-Gesztelyi for comments and suggestions, and to N. I. Bondar and I Yu. Alekseev who kindly provided their photographic observations. We are indebted to J. Bartus who carried out the data digitalization. Our referee, Prof. J. B. Rice helped us to clarify some points in the paper. $\mathrm{KO}$ and ZsK acknowledge financial support from the Hungarian government through OTKA T-026165 and T032846, through the Hungarian Space Agency grant TP 096 and from the Hungarian-French Intergovernmental grant F11/99. KGS appreciates support from the Austrian Science Foundation (FWF) grant S7301-AST. EFG acknowledges support from the National Science Foundation RUI grants AST 95-28506 and AST 00-71260.

\section{References}

Amado, P. J., Cutispoto, G., Lanza, A., \& Rodonò, M. 2000, in Proc. 11th Cambridge Workshop on Cool Stars, Stellar Systems and the Sun, ed. Garcia Lopez, Rebolo, \& Zapatero Osorio, ASP Conf. Ser., 223, CD-895

Alekseev, I. Yu, \& Bondar, N. I. 1998, private communication Barrado y Navascués, et al. 1997, A\&A, 326, 780

Bondar, N. I. 1995, A\&AS, 111, 259

Budding, E. 1977, Ap\&SS, 48, 207

Buser, R., \& Kurucz, R. L. 1992, A\&A, 264, 557

Byrne, P. B., Panangi, P. M., Lanzafame, A. C., et al. 1995, A\&A, 299, 115

Cuntz, M., Saar, S. H., \& Musielak, Z. 2000, ApJ, 533, L151

Dempsey, R. C., Linsky, J. L., Fleming, T. A., \& Schmitt, J. H. M. M. 1997, ApJ, 478, 358

Doyle, J. G., Butler, C. J., \& van den Oord, G. H. J. 1989, A\&A, 208, 208

Fang, C., \& Ding, M. D. 1995, A\&AS, 110, 99

Glebocki, R., \& Stawikowski, A. 1995, Acta Astr., 45, 725

Gleissberg, W. 1967, Solar Phys., 2, 231

Granzer, T., Schüssler, M., Caligari, P., \& Strassmeier, K. G. 2000, A\&A, 355, 1087

Griffin, R. F., Gunn, J. E., Zimmermann, B. A., \& Griffin, K. E. M. 1985, AJ, 90, 609

Güdel, M. 1992, A\&A, 264, L31

Haisch, B. M. 1989, Solar Phys., 121, 3

Halbwachs, J. L., Arenou, F., Mayor, M., Udry, S., \& Queloz, D. $2000, A \& A, 355,581$

Hartmann, L., et al. 1981, ApJ, 249, 662

Hauschildt, P., Allard, F., \& Baron, F. 1999, ApJ, 512, 377

Houdashelt, M. L., Bell., R. A., \& Sweigart, A. V. 2000, AJ, 119, 1448

Howard, R. F. 1992, Solar Phys., 137, 51

de Jager, C., Heise, J., Avgoloupis, S., \& Cutispoto, G. 1986, A\&A, 156, 95

Juckett, D. A. 2000, Solar Phys., 191, 201

Kolláth, Z. 1990, The program package MUFRAN, Occasional Technical Notes of Konkoly Observatory, No. 1 (www.konkoly.hu/Mitteilungen/Mitteilungen.html \#TechNotes)

Kővári, Zs. 1999, in Solar and Stellar Activity: Similarities and Differences, ed. C. J. Butler, \& J. G. Doyle, ASP Conf. Ser., 158,166

Kővári, Zs., \& Bartus, J. 1997, A\&A, 323, 801 
Lacy, C. H., Moffett, T. J., \& Evans, D. S. 1976, ApJS, 30, 85

Machado, M. E., Emslie, A. G., \& Avrett, E. H. 1989, Solar Phys., 124, 303

Marino, G., Rodonò, M., Leto, G., \& Cutispoto, G. 1999, A\&A, 352,189

Montes, D., Fernandez-Figueroa, M. J., de Castro, E., \& Cornide, M. 1995, A\&AS, 109, 135

Montes, D., Fernandez-Figueroa, M. J., de Castro, E., \& Sanz-Forcada, J. 1997, A\&AS, 125, 263

Naftilan, S. A., \& Fairchild, K. 1993, PASP, 105, 565

Oláh, K., \& Kolláth, Z. 1999, in Solar and Stellar Activity: Similarities and Differences, ed. C. J. Butler, \& J. G., Doyle, ASP Conf. Ser., 158, 174

Oláh, K., Kolláth, Z., \& Strassmeier, K. G. 2000, A\&A, 356, 643

Oláh, K., \& Pettersen, B. R. 1991, A\&A, 242, 443

Oláh, K., \& Strassmeier, K. G. 2000, Proc. Eleventh Cambridge Workshop on Cool Stars, Stellar Systems, and the Sun, ed. Garcia Lopez, Rebolo, \& Zapatero Osorio, ASP Conf. Ser., 223, CD-1030

Oláh, K., van Driel-Gesztelyi, L., Kővári, Zs., \& Bartus, J. 1999, A\&A, 344, 163

O'Neal, D., Neff, J. E., \& Saar, S. H. 1998, ApJ, 507, 919

O'Neal, D., Saar, S. H., \& Neff, J. E. 1996, ApJ, 463, 766

O'Neal, D., Saar, S. H., \& Neff, J. E. 1998, ApJ, 501, L73

Perryman, M. A. C., et al. 1998, A\&A, 331, 81

Pettersen, B. R. 1988, in Activity in Cool Star Envelopes, Proc. of Midnight Sun Conf., Tromso, ed. O. Havnes, B. R. Pettersen, J. H. M. M. Schmitt, \& J. E. Solheim (Kluwer), 49

Pettersen, B. R. 1989, A\&A, 209, 279
Pettersen, B. R. 1989, Solar Phys., 121, 299

Saar, S. H. 1996, in Stellar Surface Structure, Proc. of IAU Symp. No. 176, ed. K. G. Strassmeier, \& J. L. Linsky (Kluwer), 237

Saar, S. H, Golub, L., Bopp, B., Herbst, W., \& Huovelin, J. 1990, ESA SP-310, 431

Saar, S. H., Martens, P. C. H., Huovelin, J., \& Linnaluoto, S. 1994, A\&A, 286, 194

Saar, S. H., \& Neff, J. E. 1990, in Cool Stars, Stellar Systems and the Sun, Sixth Cambridge Workshop, ed. G. Wallerstein, ASP Conf Ser., 9, 171

Saar, S. H., Huovelin, J., Osten, R. A., \& Shcherbakov, A. G. 1997, A\&A, 326, 741

Saar, S. H., Peterchev, A., O'Neal, D., \& Neff, J. E. 2000, Proc. Eleventh Cambridge Workshop on Cool Stars, Stellar Systems, and the Sun, ed. Garcia Lopez, Rebolo, \& Zapatero Osorio, ASP Conf. Ser., 223, CD-1057

Strassmeier, K. G., Boyd, L. J., Epand, D. H., \& Granzer, T. 1997, PASP, 109, 697

Strassmeier, K. G., Fekel, F., Bopp, B. W., Dempsey, R. C., \& Henry, G. W. 1990, ApJS, 72, 191

Strassmeier, K. G., Bartus, J., Cutispoto, G., \& Rodonò, M. 1997, A\&AS, 125, 11

Strassmeier, K. G., Hall, D. S., Fekel, F. C., \& Scheck, M. 1993, A\&AS, 100, 173

Tokovinin, A. A. 1990, Pisma Astron. Zhurnal, 16, No. 11

Tsikoudi, V., \& Kellett, B. J. 1997, MNRAS, 285, 759

van Hamme, W. 1993, AJ, 106, 2096

Walraven, Th., Walraven, J., \& Balona, L. A. 1992, MNRAS, 254,59 